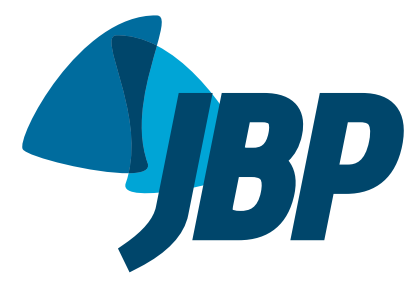

\title{
Do N-terminal pro-brain natriuretic peptide levels determine the prognosis of community acquired pneumonia?
}

\author{
Evrim Eylem Akpınar ${ }^{1, a}$, Derya Hoşgün ${ }^{2, b}$, Serdar Akpınar ${ }^{3, c}$, Can Ateș,d, \\ Ayșe Baha ${ }^{5, e}$, Esen Sayın Gülensoy ${ }^{1, f}$, Nalan Ogan ${ }^{1, \mathrm{~s}}$
}

1. Ufuk University, Faculty of Medicine, Department of Chest Diseases, Ankara, Turkey.

2. Elazıg Education and Research Hospital, Department of Intensive Care Unit, Elazıg, Turkey.

3. Diskapı Education and Research Hospital, Department of Intensive Care Unit, Ankara, Turkey.

4. Van Yuzuncu Yil University, Faculty of Medicine, Department of Biostatistics, Van, Turkey.

5. Girne Akcicek Hospital, Girne, Cyprus.

a. (D) http://orcid.org/0000-0001-9040-9309

b. (DD http://orcid.org/0000-0003-1221-3620

c. (D) http://orcid.org/0000-0003-3855-7130

d. (D) http://orcid.org/0000-0002-9649-250X

e. (D) http://orcid.org/0000-0002-0154-7775

f. (D) http://orcid.org/0000-0003-2286-4398

g. (D) http://orcid.org/0000-0001-5232-3803

Submitted: 27 December 2018

Accepted: 13 March 2019

Study carried out at Ufuk University,

Faculty of Medicine, Department of Chest Diseases, Balgat, Ankara, Turkey.

\begin{abstract}
Objective: Pneumonia is a leading cause of mortality worldwide, especially in the elderly. The use of clinical risk scores to determine prognosis is complex and therefore leads to errors in clinical practice. Pneumonia can cause increases in the levels of cardiac biomarkers such as N-terminal pro-brain natriuretic peptide (NT-proBNP). The prognostic role of the NT-proBNP level in community acquired pneumonia (CAP) remains unclear. The aim of this study was to evaluate the prognostic role of the NT-proBNP level in patients with CAP, as well as its correlation with clinical risk scores. Methods: Consecutive inpatients with CAP were enrolled in the study. At hospital admission, venous blood samples were collected for the evaluation of NT-proBNP levels. The Pneumonia Severity Index (PSI) and the Confusion, Urea, Respiratory rate, Blood pressure, and age $\geq \mathbf{6 5}$ years (CURB-65) score were calculated. The primary outcome of interest was all-cause mortality within the first 30 days after hospital admission, and a secondary outcome was ICU admission. Results: The NT-proBNP level was one of the best predictors of 30-day mortality, with an area under the curve (AUC) of 0.735 (95\% CI: 0.642-0.828; p $<0.001)$, as was the PSI, which had an AUC of 0.739 (95\% Cl: $0.634-0.843 ; p<0.001)$, whereas the CURB-65 had an AUC of only 0.659 (95\% Cl: $0.556-0.763 ; p=0.006$ ). The NT-proBNP cut-off level found to be the best predictor of ICU admission and 30day mortality was $1,434.5 \mathrm{pg} / \mathrm{mL}$. Conclusions: The NT-proBNP level appears to be a good predictor of ICU admission and 30-day mortality among inpatients with CAP, with a predictive value for mortality comparable to that of the PSI and better than that of the CURB-65 score.
\end{abstract}

Keywords: Pneumonia/diagnosis; Pneumonia/mortality; Natriuretic peptide, brain; Community-acquired infections.

\section{INTRODUCTION}

Despite recent developments in clinical care and antimicrobial treatment, pneumonia continues to be a leading cause of mortality worldwide, especially in the elderly. In cases of community-acquired pneumonia (CAP), the Pneumonia Severity Index (PSI) and the Confusion, Urea, Respiratory rate, Blood pressure, and age $\geq \mathbf{6 5}$ years (CURB-65) score are used in order to predict the severity and determine the prognosis. ${ }^{(1,2)}$ However, calculating risk scores, especially the PSI, is complex and is dependent on subjective impressions, which can lead to errors in clinical practice. ${ }^{(3)}$ Brain natriuretic peptide (BNP) is a potent natriuretic and diuretic hormone that is released from the heart into the systemic circulation and is enzymatically cleaved into active and inactive forms-BNP and N-terminal pro-brain natriuretic peptide (NT-proBNP), respectively. The optimal timing of NT-proBNP assessment for risk stratification is unclear. Weber et al.(4) showed that, to determine the peak elevation of NT-proBNP, it is necessary to perform sequential testing, the second sample being collected 24-36 $\mathrm{h}$ after the onset of symptoms of acute coronary syndrome. However, it remains an open issue whether the peak values of NT-proBNP are of superior predictive value. The levels of BNP and NT-proBNP are elevated in cases of increased myocardial strain. In patients with cardiac failure, the NT-proBNP level can be measured rapidly with a simple method. Pneumonia can also cause an increase in the levels of cardiac biomarkers. ${ }^{(5,6)}$

As compared with BNP, NT-proBNP has a number of advantages, including a longer half-life and higher in vitro stability. ${ }^{(7)}$ Although previous studies have suggested that BNP and NT-proBNP levels are useful for risk stratification and for determining the prognosis in patients with $\mathrm{CAP}_{,}{ }^{(8,9)}$ the prognostic role of the NT-proBNP level in CAP remains unclear. The aim of this study was to evaluate the prognostic role of the NT-proBNP level in patients with CAP by investigating its association with 30-day mortality, ICU admission, length of hospital stay, and clinical risk scores (PSI and CURB-65 score).

\section{METHODS}

This was a prospective study in which consecutive patients who were hospitalized for CAP between March 2014 and October 2018 were enrolled. The study protocol 
was approved by the local research ethics committee, and all participating patients gave written informed consent.

The inclusion criteria were as follows: being $\geq 18$ years of age; having a fever $\left(\geq 37.3^{\circ} \mathrm{C}\right)$; presenting with cough of recent onset, sputum production, or other symptoms of respiratory infection, including purulent sputum, with or without chest pain; having a leukocyte count $>10 \times 10^{9} / \mathrm{L}$ or $<4 \times 10^{9} / \mathrm{L}$; and showing patchy infiltrative shadows or interstitial changes, with or without effusion, on chest X-rays. Patients with active tuberculosis, lung cancer, pulmonary fibrosis, pulmonary embolisms, pulmonary hypertension, or nosocomial pneumonia were excluded, as were those with congestive heart failure, cirrhosis, acute coronary syndrome, end-stage renal disease, or acute kidney injury.

The levels of NT-pro-BNP were assessed with the Elecsys-ProBNP assay (Roche Diagnostics, Penzberg, Germany). The assay uses two polyclonal antibodies, one of which is labeled with ruthenium complex and measures the inactive cleavage product of BNP (i.e., NT-pro-BNP). It also includes an automated electrochemiluminescence immunoassay. The Elecsys immunoassay analyzer (Roche Diagnostics) provides the initial results in $18 \mathrm{~min}$. Two cut-off points are used, one at $125 \mathrm{pg} / \mathrm{mL}$, for patients < 75 years of age, and the other at $450 \mathrm{pg} / \mathrm{mL}$, for patients $\geq 75$ years of age. ${ }^{(10)}$ High-sensitivity C-reactive protein levels were measured by automatic calorimetric assay in a COBAS INTEGRA 400 plus analyzer (Roche Diagnostics, Mannheim, Germany), with a cut-off level of $5 \mu \mathrm{g} / \mathrm{L}$.

The medical histories and demographic characteristics of the patients were recorded. At admission, vital signs, complete blood counts, serum glucose levels, liver function, kidney function, and arterial blood gases were assessed. A chest X-ray was also obtained. The PSI and CURB-65 scores of the patients were calculated in accordance with the American Thoracic Society guidelines. ${ }^{(1)}$ The need for ICU admission was determined on the basis of the criteria established by the Infectious Diseases Society of America and the American Thoracic Society guidelines for CAP. ${ }^{(11)}$ Acute physiology and chronic health evaluation II (APACHE II) scores were calculated for patients admitted to the ICU. ${ }^{(12)}$

To exclude cardiac causes of myocardial strain, such as congestive heart failure, we performed electrocardiography and transthoracic echocardiography at admission. Echocardiographic measurements were performed on a ViVid 7 Pro system (GE Vingmed, Horten, Norway) with a $1.7 \mathrm{MHz}$ transducer that was capable of harmonic imaging. Conventional echocardiography and tissue Doppler imaging were performed by two cardiologists who were blinded to the clinical status of the subjects. Pulmonary hypertension was defined as a systolic pulmonary arterial pressure $>35 \mathrm{mmHg}$, and left ventricular systolic dysfunction was defined as an ejection fraction $<50 \%$. ${ }^{(13,14)}$
The primary outcome of interest was all-cause mortality within the first 30 days after hospital admission. Secondary outcomes were ICU admission and the length of the hospital stay.

\section{Statistical analysis}

The statistical analysis was performed with the SPSS Statistics software package, version 23.0 (IBM Corporation, Armonk, NY, USA). To identify the risk factors for ICU admission and 30-day mortality, we performed univariate and multivariate logistic regression analyses. In the multivariate logistic regression models, backward logistic regression was used and the criterion for inclusion of the candidate variables in the model was a $p<0.25$. Multivariate linear regression models were used for continuous response variables such as length of hospital stay. The prognostic performance of parameters such as the CURB-65, the PSI, and $\mathrm{SpO}_{2}$ was compared by using ROC curve analysis. The results are summarized as the area under the curve (AUC), with $95 \%$ confidence intervals and standard errors. In addition, the optimal cut-off points were calculated by using the Youden index. For group comparisons, normal distribution was assessed with the Shapiro-Wilk test, continuous variables were assessed with Student's t-tests or Mann-Whitney U tests, and categorical variables were assessed with chi-square tests or Fisher's exact tests. The relationships between variables such as the CURB-65 score, PSI, APACHE II score, and NT-proBNP level were examined with Spearman's correlation coefficients. Values of $p<0.05$ were considered statistically significant.

\section{RESULTS}

Initially, 179 patients were considered for inclusion in the study. On the basis of the study criteria, 24 of those patients were excluded for presenting with one of the following comorbidities: active tuberculosis ( $n=1)$; lung cancer $(n=3)$; pulmonary fibrosis $(n=2)$; pulmonary embolism $(n=2)$; pulmonary hypertension ( $n=1)$; nosocomial pneumonia $(n=2)$; congestive heart failure $(n=4)$; cirrhosis $(n=1)$; acute coronary syndrome $(n=3)$; end-stage renal disease $(n=3)$; and acute kidney injury $(n=2)$. Therefore, 155 patients were enrolled in the study. Of those 155 patients, 54 (34.8\%) were female and 101 (65.2\%) were male. The mean age of the patients was 72.70 \pm 12.64 years. The gender distribution, smoking status, PSI, CURB-65 scores, mortality rates, and ICU admission rates are shown in Table 1.

The univariate analysis showed that the factors influencing ICU admission and 30-day mortality were the PSI ( $p<0.001$ for both), CURB-65 score ( $p<$ 0.001 and $p=0.002$, respectively), serum NT-proBNP level ( $p<0.001$ and $p=0.023$, respectively), and leukocyte count ( $p=0.034$ and $p=0.024$, respectively). Multivariate logistic regression analysis showed that the PSI and CURB-65 score were both predictive of 30-day mortality. According to the univariate analysis, the factors that affected the length of hospital stay were 
the CURB-65 score, serum NT-proBNP level, albumin level, leukocyte count, and $\mathrm{SpO}_{2}(\mathrm{p}<0.001, \mathrm{p}<$ $0.001, p=0.020$, and $p=0.024$, respectively). Table 2 shows the results of the univariate and multivariate logistic regression analyses of the potential predictors of 30-day mortality.

As can be seen in Figure 1, the mean NT-proBNP level was significantly higher among the patients who died

Table 1. Gender distribution, smoking status, clinical risk scores, ICU admission rates, and mortality rates among inpatients with community-acquired pneumonia $(\mathrm{N}=155)$.

\begin{tabular}{|c|c|}
\hline Variable & n (\%) \\
\hline \multicolumn{2}{|l|}{ Gender } \\
\hline Female & $54(34.8)$ \\
\hline Male & 101 (65.9) \\
\hline \multicolumn{2}{|l|}{ Smoking status } \\
\hline Never smoker & $68(43.9)$ \\
\hline Current smoker & $23(14.8)$ \\
\hline Former smoker & $64(41.3)$ \\
\hline \multicolumn{2}{|l|}{ PSI } \\
\hline 1 & $2(1.3)$ \\
\hline 2 & $22(14.2)$ \\
\hline 3 & $72(46.5)$ \\
\hline 4 & $54(34.8)$ \\
\hline 5 & $5(3.2)$ \\
\hline \multicolumn{2}{|l|}{ CURB-65 score } \\
\hline 0 & $7(4.5)$ \\
\hline 1 & $25(16.1)$ \\
\hline 2 & $58(37.4)$ \\
\hline 3 & $56(36.1)$ \\
\hline 4 & $8(5.2)$ \\
\hline 5 & $1(0.6)$ \\
\hline \multicolumn{2}{|l|}{ ICU admission } \\
\hline Yes & $42(27.1)$ \\
\hline No & $113(72.9)$ \\
\hline \multicolumn{2}{|l|}{ 30-day mortality } \\
\hline Yes & $31(20.0)$ \\
\hline No & $124(80.0)$ \\
\hline
\end{tabular}

within the first 30 days after hospital admission than among those who survived $(4,594.41 \pm 6,993.71 \mathrm{pg} /$ $\mathrm{mL}$ vs. $1,759.98 \pm 3,589.21 \mathrm{pg} / \mathrm{mL} ; \mathrm{p}=0.002)$. The mean NT-proBNP level was also significantly higher among the patients who were admitted to the ICU than among those who were not $(5,209.50 \pm 7,807.21$ $\mathrm{pg} / \mathrm{mL}$ vs. $1,255.44 \pm 1,562.32 \mathrm{pg} / \mathrm{mL} ; \mathrm{p}<0.001)$.

When we employed Spearman's correlation coefficients to examine the simple correlations, we found a significant correlation between the NT-proBNP level and the PSI $(r=0.441 ; p<0.001)$. The PSI also correlated significantly with the CURB-65 score ( $r=$ $0.318 ; p<0.001)$. The simple correlations among the NT-proBNP level, PSI, CURB-65 score, and APACHE II score are shown in Table 3.

A ROC curve showed that the PSI, NT-proBNP level, and CURB-65 score all had predictive value for 30-day mortality. The PSI and NT-proBNP level had similar predictive values for 30-day mortality, both of which were better than that of the CURB- 65 score. The AUCs for the PSI, NT-proBNP level, and CURB-65 score, respectively, were as follows (Figure 2): 0.739 (95\% CI: $0.634-0.843$; $p<0.001)$; 0.735 (95\% CI: $0.642-$ $0.828 ; \mathrm{p}<0.001$ ); and 0.659 (95\% CI: 0.556-0.763; $p=0.006)$. The best NT-proBNP cut-off level for the prediction of ICU admission and 30-day mortality was found to be $1,434.5 \mathrm{pg} / \mathrm{mL}$, which had a sensitivity and specificity of 0.738 and 0.735 , respectively, for ICU admission, compared with 0.710 and 0.685 , respectively, for 30-day mortality.

The ROC analysis was performed with the probabilities obtained from two different logistic models for the prediction of mortality: one including the PSI only and one including the PSI and the NT-proBNP level together. The addition of the NT-proBNP level to the PSI increased the predictive value for 30-day mortality. Figure 3 shows the ROC curves constructed from the predicted probabilities of the PSI as a single variable and of the PSI in combination with the NT-proBNP level-designated PSI $+\log ($ proBNP) -for the prediction of 30-day mortality. The AUC for the PSI was 0.772 (95\% CI: $0.682-0.861)$, with an SE of $0.046(p<$ $0.001)$, whereas the AUC for the PSI + $\log ($ proBNP)

Table 2. Results of the univariate and multivariate logistic regression analyses of the potential predictors of 30-day mortality.

\begin{tabular}{lccccc}
\multicolumn{1}{c}{ Variable } & B & SE & OR & 95\% CI & p \\
Univariate analysis & & & & & \\
$\quad$ PSI & 1.317 & 0.335 & 3.733 & $1.93-7.19$ & 0.001 \\
CURB-65 score & 0.804 & 0.253 & 2.234 & $1.35-3.67$ & 0.002 \\
$\quad$ CRP & 0.219 & 0.228 & 1.245 & $0.79-1.94$ & 0.336 \\
$\quad$ Leukocyte count* & 0.000 & 0.000 & 1.000 & $1.00-1.10$ & 0.024 \\
$\quad$ NT-proBNP level** & 0.113 & 0.050 & 1.120 & $1.01-1.23$ & 0.023 \\
Multivariate analysis & & & & & 0.001 \\
$\quad$ PSI & 1.145 & 0.351 & 3.143 & $1.58-6.25$ & 0.040 \\
$\quad$ CURB-65 score & 0.550 & 0.267 & 1.733 & $1.02-2.92$ & 0.92 \\
\hline
\end{tabular}

PSI: Pneumonia Severity Index; CURB-65: mental Confusion, Urea, Respiratory rate, Blood pressure, and age $\geq 65$ years; CRP: C-reactive protein; and NT-proBNP: N-terminal pro-brain natriuretic peptide. *100 times the exact measure. ${ }^{* *} 1,000$ times the exact measure. 
variable was $0.812(95 \% \mathrm{CI}: 0.727-0.897)$, with an SE of $0.043(p<0.001)$.

\section{DISCUSSION}

The main finding of the present study was that the NT-proBNP level correlated significantly with the PSI and CURB-65 score in terms of the mean length of hospital stay and the prediction of mortality. The NT-proBNP level was one of the best predictors of mortality. The NT-proBNP level also correlated significantly with the CURB-65 score in patients admitted to the ICU. The best NT-proBNP cut-off level for the prediction of ICU admission and 30 -day mortality was $1,434.5 \mathrm{pg} / \mathrm{mL}$. The combination of the NT-proBNP level and the PSI had a better predictive value for 30-day mortality than did the PSI alone.

The PSI and CURB-65 are risk scoring systems used in evaluating the severity of CAP. However, they play a limited role in determining the prognosis of CAP. It was previously shown that the PSI and CURB-65 score both markedly underestimated mortality, particularly in the low-risk strata. That leads to the misclassification of patients with substantial mortality in the low-risk strata. ${ }^{(15)}$ The CURB-65 score and PSI have both been shown to have low predictive specificity, incorrectly categorizing many young patients as being at low risk. ${ }^{(16)}$ The present study showed that the PSI and CURB-65 score both had predictive value for mortality. Because our study population included only inpatients

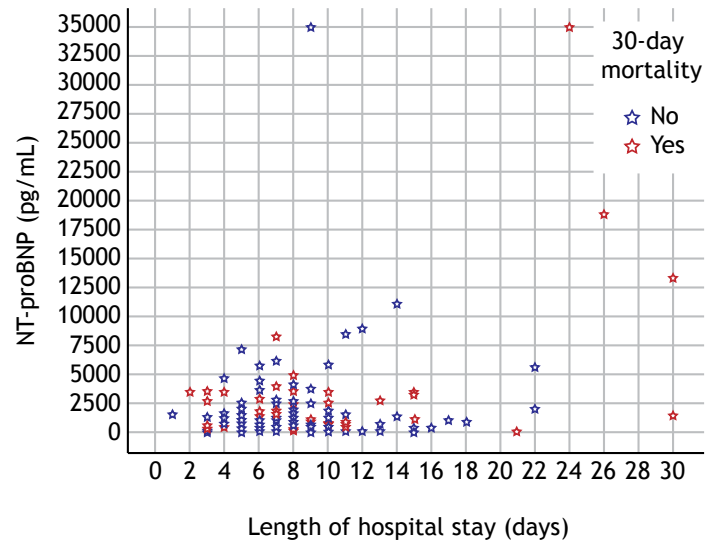

Figure 1. Levels of $\mathrm{N}$-terminal pro-brain natriuretic peptide (NT-proBNP) among patients with community-acquired pneumonia who died within the first 30 days after hospital admission and among those who survived. with CAP, most of the patients were in the high-risk strata and were elderly (mean age, 72.70 years). Those properties might have had a preventive effect against underestimation of mortality in the low-risk strata and young patients. In addition, the calculation of the PSI is complicated and its use is therefore not feasible in clinical practice, especially in the emergency department. Although the CURB- 65 scoring system is simpler than is that of the PSI, the former has lower sensitivity for the prediction of 30 -day mortality. ${ }^{(16,17)}$ In a study of the role of the BNP level in predicting the severity of CAP, Li et al. ${ }^{(18)}$ showed that it correlated positively with the severity of CAP, which is in accordance with the findings of the present study. In their study, the best BNP cut-off level for predicting mortality was found to be $299 \mathrm{pg} / \mathrm{mL}$, which had good sensitivity and even better specificity. Usuda et al. ${ }^{(8)}$ also studied the prognostic role of the BNP level in patients with pneumonia and found that a high BNP level ( $\geq 200$ $\mathrm{pg} / \mathrm{mL}$ ) at admission was a predictor of CAP-related death. ${ }^{(8)}$ In patients with cardiac or renal dysfunction, the

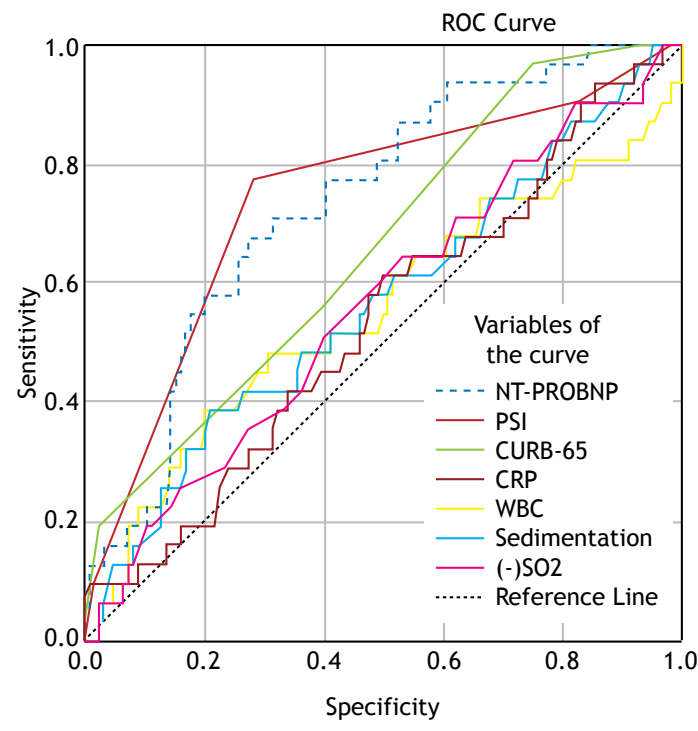

Figure 2. The ROC curve for potential predictors of 30-day mortality. The areas under the ROC curve were as follows: 0.739 (95\% CI: 0.634-0.843) for the Pneumonia Severity Index (PSI; $p<0.001) ; 0.735$ (95\% CI: 0.642-0.828) for the $\mathrm{N}$-terminal pro-brain natriuretic peptide (NT-proBNP) level ( $p<0.001)$; and 0.659 (95\% CI: $0.556-0.763$ ) for the Confusion, Urea, Respiratory rate, Blood pressure, and age $\geq \mathbf{6 5}$ years (CURB-65) score $(p=0.006)$. CRP: C-reactive protein.

Table 3. Simple correlation coefficients among selected variables.

\begin{tabular}{lccc}
\multicolumn{1}{c}{ Variable } & PSI & CURB-65 score & APACHE II score \\
& Spearman's rho $(p)$ & $0.086(0.286)$ & $0.113(0.475)$ \\
NT-ppearman's rho $(p)$ & $0.241(0.124)$ \\
PSI & $0.441(<0.001)$ & $0.318(<0.001)$ & $0.103(0.514)$ \\
CURB-65 score & - & - & - \\
APACHE II score & & & \\
\hline
\end{tabular}

NT-proBNP: N-terminal pro-brain natriuretic peptide; PSI: Pneumonia Severity Index; CURB-65: mental Confusion, Urea, Respiratory rate, Blood pressure, and age $\mathbf{2} \mathbf{6 5}$ years; and APACHE II: Acute Physiology and Chronic Health Evaluation II. 


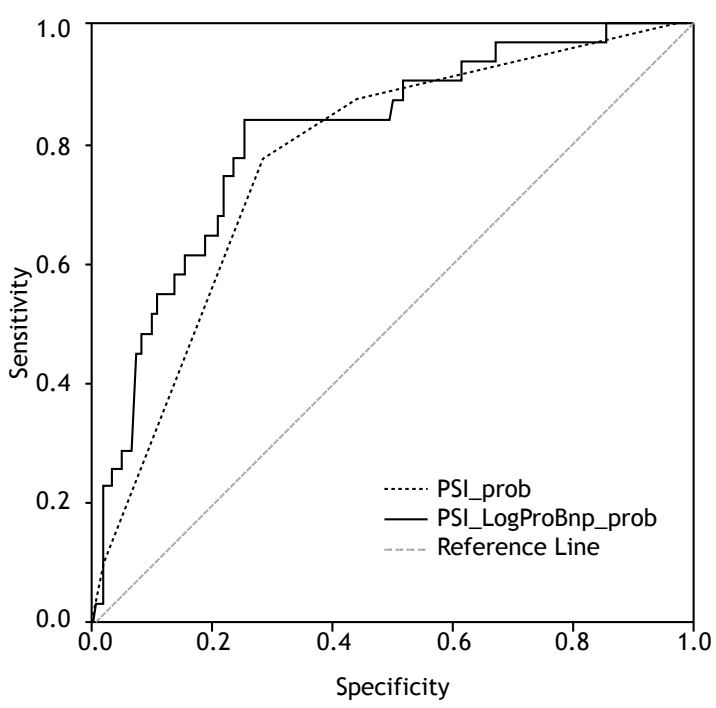

Figure 3. The ROC curves constructed from the predicted probabilities of the PSI as a single variable and of the PSI in combination with the NT-proBNP level-designated PSI $+\log ($ proBNP) - for the prediction of 30-day mortality. The AUC for the PSI was 0.772 (95\% CI: 0.682-0.861), with an SE of $0.046(p<0.001)$, whereas the AUC for the PSI $+\log$ (proBNP) variable was 0.812 (95\% CI: 0.727-0.897), with an SE of $0.043(p<0.001)$.

level of NT-proBNP rises more steeply than does that of BNP. Furthermore, in comparison with BNP, NT-proBNP is larger, is detected more rapidly, is more biologically stable, and has a longer half-life. ${ }^{(19,20)}$ Therefore, we opted to measure NT-proBNP, rather than BNP, in the present study. As previously mentioned, we found the best NT-proBNP cut-off level for the prediction of ICU admission and 30-day mortality to be 1,434.5 $\mathrm{pg} / \mathrm{mL}$, which had good sensitivity and specificity for both outcome measures.

Jeong et al. ${ }^{(21)}$ showed that the NT-proBNP levels of patients with CAP who died were significantly higher than were those of survivors. ${ }^{(21)}$ Our results support that finding, the NT-proBNP levels of our patients who died within the first 30 days after hospital admission being significantly higher than were those of the patients who survived. We also evaluated the relationship between ICU admission and NT-proBNP levels, finding that the NT-proBNP levels of the patients who were admitted to the ICU were significantly higher than were those of the patients who did not require ICU admission.

Lin et al. ${ }^{(22)}$ investigated the plasma levels of NT-proBNP at ICU admission and 30-day mortality in patients with pneumonia, healthcare-associated pneumonia accounting for $40 \%$ of the sample, whereas CAP and hospital-acquired pneumonia accounted for $35 \%$ and $25 \%$, respectively. Those authors also found that the mean NT-proBNP levels were significantly lower among the survivors than among the nonsurvivors. They also reported that the NT-proBNP level showed prognostic accuracy comparable to that of the APACHE II score in patients who were admitted to the ICU with pneumonia. However, although the NT-proBNP levels correlated significantly with the CURB-65 score in patients admitted to the ICU in the present study, there was no correlation between those levels and the APACHE II scores in those same patients.

Nowak et al.(9) suggested that the levels of natriuretic peptides, especially NT-proBNP, could predict mortality in CAP and that their predictive ability is comparable to that of the PSI. ${ }^{(9)}$ Consistent with that idea, we found that the predictive value of the NT-proBNP level was comparable to that of the PSI and that the former was a more powerful predictor than was the CURB-65 score. Although the PSI is a validated tool, it includes many parameters related to demographic characteristics, comorbidities, and laboratory findings, as well as being partially dependent on subjective impressions. In contrast, the measurement of the NT-proBNP level is simple and objective. Therefore, it could be an alternative to the PSI for predicting the severity and determining the prognosis of CAP. In addition, our study showed that combining the NT-proBNP level and the PSI increased the predictive value for 30-day mortality over that of the PSI alone.

The mechanism of myocardial strain, which increases the levels of cardiac biomarkers in patients with pneumonia, is not clear. Musher et al.(23) found a myocardial infarction rate of $7-8 \%$ among patients who were hospitalized for pneumonia. The risk of myocardial infarction associated with pneumonia peaks at the onset of infection and is proportional to the severity of the pneumonia. ${ }^{(24)}$ In the present study, we excluded patients who had comorbidities that can affect the NT-proBNP level, such as acute coronary syndrome, in order to avoid the confounding effects of such comorbidities. CAP places significant stress on the cardiovascular system via the induction of low peripheral vascular resistance, increased cardiac output, and the occurrence of arteriovenous shunts in areas of inflammation. (9) Studies in patients with sepsis have suggested that cardiac biomarker levels reflect the extent of systemic inflammation. ${ }^{(23,25)}$ Zhang et al.(26) found that the NT-proBNP levels of patients with pneumonia correlated positively with inflammatory markers, such as the leukocyte count, ESR, and C-reactive protein level. Among the biochemical parameters evaluated in the present study, the consistent predictors of ICU admission and 30-day mortality included not only the NT-proBNP level but also the leukocyte count. However, we did not detect any relationships between mortality and other inflammatory markers, such as the C-reactive protein level and the ESR.

The present study has several limitations. First, the study population consisted only of inpatients with CAP, most of whom were elderly. In addition, patients who had comorbidities that could affect the NT-proBNP level were excluded from the study. Therefore, it might not be possible to extrapolate our findings to the general population of patients with CAP. Second, this was a single-center study conducted at a university hospital. Multicenter studies of patients with CAP might help clarify the prognostic role of the NT-proBNP level. 
In conclusion, the NT-proBNP level appears to be a good predictor of ICU admission and short-term mortality among patients hospitalized for CAP. We found its predictive value for mortality to be comparable to that of the PSI and better than that of the CURB-65 score. We also found that the inclusion of the NT-proBNP level increased the predictive value of PSI for mortality. There is a need for population-based randomized controlled studies in order to determine the exact prognostic role that the NT-proBNP level plays in CAP and to validate NT-proBNP as a prognostic marker in the general population of patients with CAP.

\section{REFERENCES}

1. Niederman MS, Mandell LA, Anzueto A, Bass JB, Broughton WA Campbell GD, et al. Guidelines for the management of adults with community-acquired pneumonia: diagnosis, assessment of severity, antimicrobial therapy, and prevention. Am J Respir Crit Care Med. 2001;163(7):1730-54. https://doi.org/10.1164/ajrccm.163.7.at1010

2. Piffer F, Tardini F, Cosentini R. The IDSA/ATS CAP consensus guidelines on the management of CAP in adults. Breathe. 2007:4(2):111-115

3. Aronsky D, Haug PJ. Assessing the quality of clinical data in a computer-based record for calculating the pneumonia severity index. J Am Med Inform Assoc. 2000;7(1):55-65. https://doi.org/10.1136/ jamia.2000.0070055

4. Weber M, Kleine C, Keil E, Rau M, Berkowitsch A, Elsaesser A, et al. Release pattern of $\mathrm{N}$-terminal pro B-type natriuretic peptide (NT-proBNP) in acute coronary syndromes. Clin Res Cardiol. 2006;95(5):270-80. https://doi.org/10.1007/s00392-006-0375-x

5. Dickstein K, Cohen-Solal A, Filippatos G, McMurray JJ, Ponikowski P. Poole-Wilson PA, et al. ESC Guidelines for the diagnosis and treatment of acute and chronic heart failure 2008: the Task Force for the Diagnosis and Treatment of Acute and Chronic Heart Failure 2008 of the European Society of Cardiology. Developed in collaboration with the Heart Failure Association of the ESC (HFA) and endorsed by the European Society of Intensive Care Medicine (ESICM). Eur Heart J. 2008;29(19):2388-442

6. de Lemos JA, McGuire DK, Drazner MH. B-type natriuretic peptide in cardiovascular disease. Lancet. 2003;362(9380):316-22. https://doi. org/10.1016/S0140-6736(03)13976-1

7. Ordonez-Llanos J, Collinson PO, Christenson RH. Amino-terminal pro-B-type natriuretic peptide: analytic considerations. Am J Cardiol. 2008;101(3A):9-15. https://doi.org/10.1016/j.amjcard.2007.11.013

8. Usuda D, Sangen R, Hashimoto Y, Muranaka E, linuma Y, Kanda T. Validation of a B-type natriuretic peptide as a prognostic marker in pneumonia patients: a prospective cohort study. BMJ Open. 2016;6(2):e010440. https://doi.org/10.1136/bmjopen-2015-010440

9. Nowak A, Breidthardt T, Christ-Crain M Bingisser R, Meune C Tanglay $Y$, et al. Direct comparison of three natriuretic peptides for prediction of short- and long-term mortality in patients with community-acquired pneumonia. Chest. 2012;141(4):974-982. https://doi.org/10.1378/chest.11-0824

10. Elecsys NT-pro-BNP Package Insert, revision 2002-11. Mannheim: Roche Diagnostics GmbH; 2011.

11. Mandell LA, Wunderink RG, Anzueto A, Bartlett JG, Campbell GD Dean NC, et al. Infectious Diseases Society of America/American Thoracic Society consensus guidelines on the management of community-acquired pneumonia in adults. Clin Infect Dis. 2007;44 Suppl 2:S27-72. https://doi.org/10.1086/511159

12. Knaus WA, Zimmerman JE, Wagner DP, Draper EA, Lawrence DE. APACHE-acute physiology and chronic health evaluation: a physiologically based classification system. Crit Care Med. 1981;9(8):591-7. https://doi.org/10.1097/00003246-19810800000008

13. Hurrell DG, Nishimura RA, Ilstrup DM, Appleton CP. Utility of preload alteration in assessment of left ventricular filling pressure by Doppler echocardiography: a simultaneous catheterization and Doppler echocardiographic study. J Am Coll Cardiol. 1997;30(2):459-67. https://doi.org/10.1016/S0735-1097(97)00184-8

14. Nishimura RA, Tajik AJ. Evaluation of diastolic filling of left ventricle in health and disease: Doppler echocardiography is the clinician's Rosetta Stone. J Am Coll Cardiol. 1997;30(1):8-18. https://doi. org/10.1016/S0735-1097(97)00144-7

15. Schuetz P, Koller M, Christ-Crain M, Steyerberg E, Stolz D, Muller $\mathrm{C}$, et al. Predicting mortality with pneumonia severity scores: importance of model recalibration to local settings. Epidemiol Infect. 2008;136(12):1628-37. https://doi.org/10.1017/S0950268808000435

16. Liu JL, Xu F, Zhou H, Wu XJ, Shi LX, Lu RQ, et al. Expanded CURB65: a new score system predicts severity of community-acquired pneumonia with superior efficiency. Sci Rep. 2016;6:22911. https:// doi.org/10.1038/srep22911

17. Ewig S, de Roux A, Bauer T, García E, Mensa J, Niederman M et al. Validation of predictive rules and indices of severity for community acquired pneumonia. Thorax. 2004;59(5):421-7. https:// doi.org/10.1136/thx.2003.008110

18. LiJ, Ye H, Zhao L. B-type natriuretic peptide in predicting the severity of community-acquired pneumonia. World J Emerg Med. 2015;6(2):131 6. https://doi.org/10.5847/wjem.j.1920-8642.2015.02.008

19. Boomsma F, van den Meiracker AH. Plasma A- and B-type natriuretic peptides: physiology, methodology and clinical use. Cardiovasc Res. 2001;51(3):442-9. https://doi.org/10.1016/S0008-6363(01)00195-X

20. Masson S, Vago T, Baldi G, Salio M, De Angelis N, Nicolis E, et al. Comparative measurement of $\mathrm{N}$-terminal pro-brain natriuretic peptide and brain natriuretic peptide in ambulatory patients with heart failure. Clin Chem Lab Med. 2002;40(8):761-3. https://doi. org/10.1515/CCLM.2002.130

21. Jeong KY, Kim K, Kim TY, Lee CC, Jo SO, Rhee JE, et al. Prognostic value of $\mathrm{N}$-terminal pro-brain natriuretic peptide in hospitalised patients with community-acquired pneumonia Emerg Med J. 2011;28(2):122-7. https://doi.org/10.1136/emj.2009.089383

22. Lin SC, Tsai YJ, Huang CT, Kuo YW, Ruan SY, Chuang YC, et al Prognostic value of plasma $\mathrm{N}$-terminal pro B-type natriuretic peptide levels in pneumonia patients requiring intensive care unit admission. Respirology. 2013;18(6):933-41. https://doi.org/10.1111/resp.12096

23. Musher DM, Abers MS, Corrales-Medina VF. Acute Infection and Myocardial Infarction. N Engl J Med. 2019;380(2):171-176. https:// doi.org/10.1056/NEJMra1808137

24. Shor R, Rozenman $Y$, Bolshinsky A, Harpaz D, Tilis $Y$, Matas Z, et al. BNP in septic patients without systolic myocardial dysfunction. Eur J Intern Med. 2006;17(8):536-40. https://doi.org/10.1016/j. ejim.2006.07.013

25. Maeder M, Ammann P, Kiowski W, Rickli H. B-type natriuretic peptide in patients with sepsis and preserved left ventricular ejection fraction. Eur J Heart Fail. 2005;7(7):1164-7. https://doi.org/10.1016/j. ejheart.2005.03.003

26. Zhang $S$, Zhang $H X$, Lin RY, Zhang SM, Xuet ZY. Predictive role of NT-pro BNP for adverse cardiac events in communityacquired pneumonia: a retrospective study. Int J Clin Exp Med. 2016;9(7):14411-14417. 